nuclear receptors. SRC-1, for example, contains four LXXLL motifs, which are essential for its interaction with the oestrogen receptor, and to mediate the induction of target genes in a ligand-dependent manner ${ }^{2}$.

The involvement of leucine-rich motifs in the assembly of nuclear-factor complexes is not new. Leucine-zipper domains, for instance, have been shown to mediate dimerization of nuclear factors such as jun, fos and CREB, through a heptad repeat of leucine residues $^{17}$. However, not all of the proteins that contain leucine zippers can form heterodimers with one another - partner specificity is determined by the presence of intervening polar residues, which form salt bridges to further stabilize homo- and heterodimer formation ${ }^{17}$.

Torchia et al. and Heery et al. support the notion that, in a similar manner, the residues within the LXXLL motifs may determine which nuclear receptors assemble with which co-activators following hormonal induction. An acidic amino acid at position 3 within the LXXLL motif, for example, seems to be particularly unfavourable for interaction with the oestrogen receptor ${ }^{2}$. Notably, CBP contains an (acidic) glutamate residue at position 3 of its LXXLL motif, and seems to bind the AF2 domain of the oestrogen receptor far less efficiently than a motif in SRC-1 which contains a (basic) arginine residue at the same position ${ }^{2}$. Given the apparently striking differences in the affinity of the oestrogen receptor for binding CBP and SRC1, it will be important to work out which of these related motifs are used by individual nuclear-receptor family members in vivo.

Our understanding of the process by which steroid hormones and other extracellular signals stimulate target-gene expression is far from complete. But the description of a short motif that mediates the formation of complexes between nuclear receptors and these co-activators greatly simplifies that endeavour, by allowing for the eventual development of antagonists that can disrupt these complexes.

Marc Montminy is in the Section on Molecular Biology, Joslin Diabetes Center, One Joslin Place,

Boston, Massachusetts 02215, USA.

1. Torchia, J. et al. Nature 387, 677-684 (1997).

2. Heery, D. M., Kalkhoven, E., Hoare, S. \& Parker, M. G. Nature 387, 733-736 (1997).

3. Tsai, M. J. \& O'Malley, B. W. Annu. Rev. Biochem. 63, 451-486 (1994).

4. Danielian, P. S., White, R., Lees, J. A. \& Parker, M. G. EMBO J. 11, 1025-1033 (1992)

5. Durand, B. et al. EMBO J. 13, 5370-5382 (1994).

6. Onate, S. A., Tsai, S. Y., Tsai, M. J. \& O'Malley, B. W. Science 270, 1354-1357 (1995).

7. Voegel, J. J., Heine, M. J. S., Zechel, C., Chambon, P. \& Gronemeyer, H. EMBO J. 15, 3667-3675 (1996).

8. Kwok, R. P. et al. Nature 370, 223-226 (1994).

9. Arias, J. et al. Nature 370, 226-229 (1994).

10. Kamei, Y. et al. Cell 85, 403-415 (1996).

11. Chakravarti, D. et al. Nature 383, 99-103 (1996).

12. Ogryzko, V. V., Shiltz, R. L., Russanova, V., Howard, B. H. \& Nakatani, Y. Cell 87, 953-960 (1996).

13. Bannister, A. J. \& Kouzarides, T. Nature 384, 641-643 (1996).

14. Nakajima, T., Uchida, C., Anderson, S. F., Parvin, J. D. \& Montminy, M. Genes Dev. 11, 738-747 (1997).

15. Yao, T. P., Ku, G., Zhou, N., Scully, R. \& Livingston, D. M. Proc. Natl Acad. Sci. USA 93, 10626-10631 (1996).

16. Smith, C. L., Onate, S. A., Tsain, M. J. \& O’Malley, B. W. Proc. Natl Acad. Sci. USA 93, 8884-8888 (1996).

17. Landschulz, W. H., Johnson, P. F. \& McKnight, S. L. Science 240, 1759-1764 (1988).

\title{
The restless heart of a spiral
}

\section{Arun V. Holden}

$\mathrm{R}^{\mathrm{o}}$ otating spiral waves occur in a variety of two-dimensional systems that all share the property of excitability. A spiral wave has wave-like and particle-like properties — it pervades the medium and has a phase, but it also has a position, given by the coordinates of its tip. The motion of a spiral wave ${ }^{1}$, defined by motion of its tip, is called meander. This is not just a mathematical curiosity: the potentially lethal re-entrant cardiac arrhythmias of flutter and fibrillation, for example, in which the same wave of excitation repeatedly re-invades the same piece of tissue, can be modelled as spiral waves. Meander is a mechanism by which such arrhythmias can stop; and the artificial control of meander offers a means of low-voltage cardiac defibrillation, by pushing the wave tip out of the heart. At a meeting in $\mathrm{May}^{*}$, an emerging mathematical understanding of meander was described that uses distance-preserving symmetries to simplify the analysis.

*Spiral Waves, Euclidean Symmetries and Cardiac Arrhythmia

Warwick, UK, 12 May 1997.
A full mathematical description of waves in an excitable medium requires the solution of a partial differential equation, in which the variables change continuously with space and time over the whole plane. A rigidly rotating spiral wave is a time-periodic solution, whose wavefront has a shape close to that of an archimedean spiral, and the outward propagation of the wavefront produces the rotation of the spiral. The tip of a rigidly rotating spiral propagates around a circular core, within which all is at rest. If the tip is not following a simple circle (see Fig. 1), the spiral wave is distorted.

If you rotate, reflect or translate a spiral, you still have a spiral. These distance-preserving transformations of the plane are the symmetries of the euclidean group $\mathrm{E}(2)$. Based on these symmetries, Barkley ${ }^{2}$ introduced ad hoc an ordinary differential equation that describes spiral wave motion simply as motions of the tip (instead of variations over the whole plane). Solutions of this equation undergo a 'Hopf bifurcation', from a motion with a single frequency (rigid rotation) to periodic motion with two frequencies (rotation plus meander). At resonance - that is, when the two frequencies are equal - the spiral follows a superposition of a linear drift and rigid rotation. The behaviour of this system closely matches that of spiral wave dynamics obtained as solutions of the full partial differential system $^{2}$, and seen in experiments ${ }^{3}$. Furthermore, the approach naturally leads to using external periodic perturbations (in the heart, an electrical signal) to induce a controlled movement of the spiral by resonant drift ${ }^{4}$.

But to confidently design a method for controlling cardiac arrhythmias in this way, we must do better than an ad-hoc model: a rigorous mathematical framework is needed to define the range of behaviours over which the method can be applied. A rigorous derivation means reducing the system so that its motion can be separated into a superposition of motion that preserves the $\mathrm{E}(2)$ symmetries (spiral properties), and motion 'across' the group - the tip meander, which lacks these symmetries.

Although such a reduction is routine for ordinary differential systems, there are severe difficulties in such an approach to the partial differential equations that generate meandering spiral waves. These formidable technical problems have now been resolved (C. Wulff, Univ. Berlin). A rigorous proof of the necessary reduction theorem has been achieved ${ }^{5}$, bifurcations of the reduced differential equations have been studied ${ }^{6,7}$, and the evolution of single and multi-armed spirals in physical space explained ${ }^{7}$.

A simple geometrical interpretation of these rigorous results can be made for singlearmed spiral waves $^{8}$ (V. N. Biktashev, Univ. Leeds). The complicated dance of a meandering or drifting spiral wave can be simplified by moving to a rotating frame of reference attached to the tip of the spiral. This

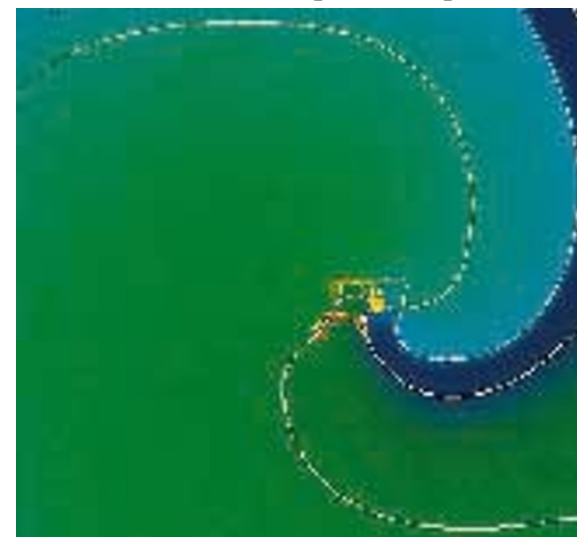

Figure 1 Visualization of the spiral wave solution of the mammalian ventricular tissue model (reproduced from ref. 11). The white lines are voltage and $\mathrm{Ca}^{2+}$ activation variable isolines; the yellow ball defines the tip position by their intersection. The orange-yellow curves map the meandering tip trajectory over the two preceding rotations of the spin. 
gives a system of ordinary differential equations describing the tip trajectory, or meander, with the frame of reference preserving the spiral nature and euclidean symmetries.

So Barkley's model system has been derived rigorously. But the heart is a threedimensional object with a complicated and moving geometry. Re-entrant waves are not spirals with a point tip, but scrolls, wrapped around a line filament. This filament can be curved; closed, as in a scroll ring, or open; twisted due to rotational anisotropy; and even under tension. Now that we have a rigorous basis for spiral wave motion, we can extend the analysis to a three-dimensional medium and consider motions of scroll waves that are possible within the $\mathrm{E}(3)$ symmetry group ${ }^{9}$ (P. Ashwin, Univ. Surrey). For example, a simple scroll can rotate and drift, whereas a twisted scroll can only rotate, and a double scroll has to remain stationary. A simple scroll ring can drift along its axis, or expand.

Such motions are seen in numerical solutions of three-dimensional caricatures (sim- plified models) of cardiac tissue, and are believed to occur during ventricular tachycardias ${ }^{10}$. Once a rigorous three-dimensional mathematical description of these excitations is available, we may finally be able to control real disturbances in the heart.

Arun V. Holden is at the Centre for Nonlinear Studies, University of Leeds, Leeds LS2 9JT, UK. e-mail:arun@cbiol.leeds.ac.uk

1. Holden, A.V., Markus, M. \& Othmer, H. G. (eds) Nonlinear Wave Phenomena in Excitable Media (Plenum, New York, 1991).

2. Barkley, D. Phys. Rev. Lett. 72, 164-167 (1994).

3. Li, G., Ouyang, Q., Petrov, V. \& Swinney, H. L. Phys. Rev. Lett. (in the press).

4. Mantel, R. M. \& Barkley, D. Phys. Rev. E 54, 4791-4801 (1996)

5. Sandstede, B., Scheel, A. \& Wulff, C. J. Diff. Eq. (in the press).

6. Fiedler, B., Sandstede, B., Scheel, A. \& Wulff, C. Doc. Math. J. DMV 1, 479-505 (1996).

7. Golubitsky, M., LeBlanc, V. G. \& Melbourne, I. J. Nonlinear Sci. (in the press).

8. Biktashev, V. N., Holden, A.V. \& Nikolaev, E. V. Int. J. Bifurcation Chaos 6, 2433-2440 (1996).

9. Ashwin, P. \& Melbourne, I. Nonlinearity 10, 595-616 (1997).

10. Panfilov, A. V. \& Holden, A. V. (eds) Computational Biology of the Heart (Wiley, Chichester, 1997).

11. Biktashev, V. N. \& Holden, A. V. Proc. R. Soc. Lond. B 263, 1373-1382 (1996).

\section{The ends of understanding}

\section{Paul Nurse}

f we had a complete description of all the molecular reactions occurring within a living cell, would we 'understand' that cell? Scientists seldom give much thought to such questions, and so it was an unusual gathering that assembled last month ${ }^{\star}$ to discuss how far a reductionist approach can take biology. Reductionism seeks to explain the wide variety of natural phenomena by the behaviour of limited numbers of simpler constituents subject to rigorous and simple laws. It has been a powerful driving force in science and its success is plainly evident in the spectacular triumphs of molecular biology which have provided an understanding of the molecular basis of areas such as developmental biology, immunology and human disease.

However, as the sole philosopher present argued (T. Nagel, NYU Law School), there are limitations to the reductionist programme. It is unlikely to be useful to try and explain biological phenomena in terms of particle physics, even if they could be so reduced. There are other levels such as molecules, genes, cells, organisms and populations which are more appropriate for adequate explanations. But what, then, is meant by an 'adequate explanation', and what is the most appropriate level to which a phenomenon should be reduced? Moreover, some generalizations and theories developed in biology may require principles that are additional to, but not inconsistent with, physical laws. Examples are concepts such as a gene, a

* The Limits of Reductionism in Biology, Ciba Foundation, London, UK, 12-15 May 1997. wing or an instinct, and the theory of natural selection - these centre on function and purpose, which are only meaningful in biology, and not physics or chemistry.

One straightforward point that strikes me is the importance of using appropriate methodologies to explain the phenomenon of interest. Thus, when reducing phenomena of cell biology to molecules and their interactions, methodologies should be employed which study molecules in the real time and space of the living cell, otherwise much of the richness of cell behaviour may be lost. For example, growth-factor signalling is often described in terms of a simple on/off device, but important information can be conveyed in the dynamics of a signalling pathway in a manner analogous to the communication of messages by the sequence and duration of signals in Morse code. The various steps of a pathway could be represented as information-processing elements, such as amplifiers, timers or integrators, as is found in an electronic circuit, rather than in terms of molecular interactions. With such representations, the same machine logic as the process under study should be used, otherwise the modelling is merely a simulation rather than an explanation. However, the detail of the molecular interactions do need to be fully described when investigating or treating a pathology (K. Holmes, MPI für Medizinische Forschung, Heidelberg), although there can be difficulties if the whole process is not also considered - for example, treatment of cardiac arrhythmias using drugs that interfere with specific molecular activities requires understanding of how the heart behaves as a whole (D. Noble, Univ. Oxford).

The importance of information processing in biology received further emphasis with the proposal that living organisms should be considered as analog computing machines which store information in the form of molecular structures (S. Brenner, Molecular Sciences Research Inst., La Jolla). Cells should not be considered as algorithmic calculating devices, but rather as a set of look-up tables from which information can be retrieved. Thus, the information specifying the Michaelis constant, $K_{\mathrm{m}}$, of an enzyme is 'stored' in the protein structure which in turn is encoded within the structure of DNA, the ultimate repository of information. The mapping of phenotypes to specific genes is equivalent to realizing the programme encoded by the DNA, but such mapping is often very complex because it depends on the actions of other interacting genes and on the past history and environment of the organism.

There are especial difficulties with such mapping in behavioural studies. The proper study of mental processes requires consideration of the products of minds and of the interactions between minds ( $\mathrm{H}$. Barlow, Univ. Cambridge). These processes are not easily reducible to cellular and molecular behaviours. For instance, it could be imagined that the recognition of 'mother' by a chick may result in the stimulation of a specific set of ten particular neurons. If these neurons are cultured in a Petri dish and then treated in a way that mimics mother recognition, would these cells in any way be experiencing the concept of mother? This seems particularly absurd. In a similar vein, can the concept of being in love be made explicable in terms of neuronal activity? It is evident that appropriate understanding needs explanation at the appropriatelevel. Likewise in studies of evolution there has been a vigorous debate over the units of selection, including arguments for group selection, inclusive fitness and gene-centred approaches (J.Maynard Smith, Univ. Sussex).

What conclusions emerged from the meeting? Unsurprisingly, there was broad support for a pragmatic pluralist approach — that is, the study of phenomena at a variety of levels. Explanations in science must always have some elements of reductionism, but descriptions of increasing detail may only provide a delusion of understanding; overenthusiastic pursuit of reductionism can limit discovery and also has ideological implications in defining what is considered to be the best science in terms of publication and financial support (S. Rose, Open Univ., Milton Keynes). But a real problem that remains is what constitutes an 'adequate explanation'. Can this ever be more than a matter of individual taste?

Paul Nurse is at the Imperial Cancer Research Fund, PO Box 123, 44 Lincoln's Inn Fields, London WC2A $3 P X, U K$. 